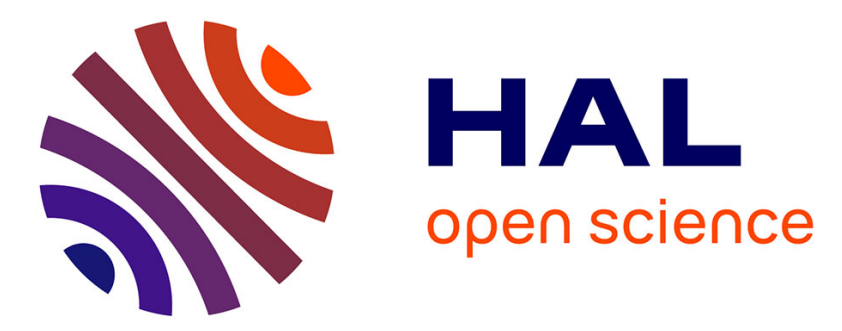

\title{
Evaporation with sodium chloride crystallization in a capillary tube
}

\author{
Antoine Naillon, Paul Duru, Manuel Marcoux, Marc Prat
}

\section{To cite this version:}

Antoine Naillon, Paul Duru, Manuel Marcoux, Marc Prat. Evaporation with sodium chloride crystallization in a capillary tube. Journal of Crystal Growth, 2015, vol. 422, pp. 52-61. 10.1016/j.jcrysgro.2015.04.010 . hal-01330778

\section{HAL Id: hal-01330778 https://hal.science/hal-01330778}

Submitted on 13 Jun 2016

HAL is a multi-disciplinary open access archive for the deposit and dissemination of scientific research documents, whether they are published or not. The documents may come from teaching and research institutions in France or abroad, or from public or private research centers.
L'archive ouverte pluridisciplinaire HAL, est destinée au dépôt et à la diffusion de documents scientifiques de niveau recherche, publiés ou non, émanant des établissements d'enseignement et de recherche français ou étrangers, des laboratoires publics ou privés. 


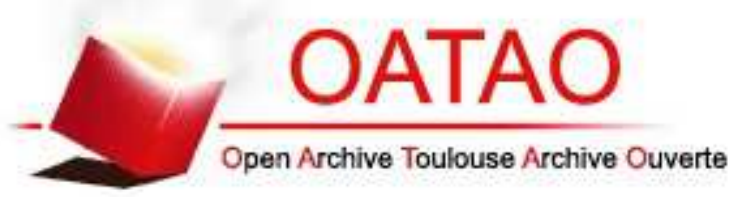

\section{Open Archive TOULOUSE Archive Ouverte (OATAO)}

OATAO is an open access repository that collects the work of Toulouse researchers and makes it freely available over the web where possible.

This is an author-deposited version published in: http://oatao.univ-toulouse.fr/ Eprints ID : 15931

To link to this article : DOI:10.1016/j.jcrysgro.2015.04.010

URL : http://dx.doi.org/10.1016/j.jcrysgro.2015.04.010

\section{To cite this version :}

Naillon, Antoine and Duru, Paul and Marcoux, Manuel and Prat, Marc Evaporation with sodium chloride crystallization in a capillary tube. (2015) Journal of Crystal Growth, vol. 422. pp. 5261. ISSN 0022-0248

Any correspondence concerning this service should be sent to the repository administrator: staff-oatao@ listes-diff.inp-toulouse.fr 


\title{
Evaporation with sodium chloride crystallization in a capillary tube
}

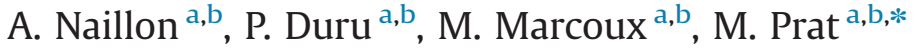 \\ a Université de Toulouse, INPT, UPS, IMFT, (Institut de Mécanique des Fluides de Toulouse), Allée Camille Soula, 31400 Toulouse, France \\ ${ }^{\mathrm{b}}$ CNRS, IMFT, 31400 Toulouse, France
}

Keywords:

A1. Evaporation

A1. Crystallization

A1. Supersaturated solution

A1. Diffusion

B1. Sodium Chloride

\begin{abstract}
A B S T R A C T
Sodium chloride crystallization induced by evaporation of aqueous solution is studied from visualization experiments in a circular capillary tube. In agreement with recent studies, the onset of crystallization is observed with a significant supersaturation. Detailed information on the precipitation kinetics and transport of ions is obtained from numerical computations of the ion mass fraction field during the evaporation process. It is shown that the precipitation kinetics is fast compared to transport so that the crystal growth is mostly controlled by the transport. This offers possible simplifications for analyzing more involved situations such as crystallization in porous media. The fact that the significant supersaturation does not lead to tube damage is explained.
\end{abstract}

\section{Introduction}

Capillary tubes can be seen as model systems for the study of porous media, [1,2]. For instance, a single capillary tube can often be considered as the elementary component in pore network models, e.g. [3]. Studies with an elementary geometry such as capillary tube are also of interest for the domain of microfluidics, e. g. [4]. In this context, the present article is devoted to a study of evaporation of a sodium-chloride aqueous solution from a single capillary tube of circular cross section.

The study of evaporation in a porous medium in the presence of a dissolved salt with possible crystallization has been the subject of several studies in recent years, e.g. [5-8] and references therein. A motivation is the study of damage on porous stones and other building materials that can result from the crystallization process, e.g. [9]. As reported in [9], the so-called crystallization pressure, which is the key-concept to analyze the generation of stresses due to the growth of crystal in pores, is directly dependent on the supersaturation at the onset of crystallization, i.e. the amount of ions in excess compared with a solution in equilibrium with salt crystals. In this paper, the supersaturation, denoted by $S$, is defined by:

$S=\frac{m o_{c r}}{m o_{s a t}}$

* Corresponding author at: IMFT, Allée Camille Soula, 31400 Toulouse, France. Tel.: + 33534322883 .

E-mail address: mprat@imft.fr (M. Prat). where $m o_{c r}$ is the molality (moles of solute by unit mass of solvent) when crystallization occurs and $\mathrm{mo}_{\text {sat }}$ is the molality at saturation $\left(m_{\text {sat }}=6.1 \mathrm{M}\right.$ for $\left.\mathrm{NaCl}\right)$.

It should be pointed out that the question of supersaturation in sodium chloride aqueous solutions is somewhat controversial. Until recently, it was widely admitted that supersaturation was negligible with $\mathrm{NaCl}$, i.e. $S \sim 1$. This was notably supported by experiments where the ion concentrations during the evaporation process were measured using NMR, i.e. [10-13]. By contrast, the experiments with capillary tubes reported in [14], indicated supersaturation as high as 1.6, a level of supersaturation largely sufficient to explain for instance the damages on porous rocks observed in the experiments reported in [15]. A significant supersaturation was also indirectly reported from the analysis of drying experiment with packings of glass beads, [5]. Owing to the crucial impact of supersaturation on stress generation, it is important to evaluate the supersaturation. One major objective of the present experiment is therefore to confirm the findings reported in [5] and [14] regarding the level of supersaturation that can be expected from evaporation of a sodium chloride aqueous solution. As reported in [16] and [13], the supersaturation can be quite significantly increased by performing delisquescence/recrystallization cycles. Here, we do not perform such cycles and are only interested in the basic situation where the solution evaporates from the capillary tube without previous purification of the solution through deliquescence/recrystallization cycles. We note that the estimate of supersaturation reported in [14] was obtained from a global mass balance (details are given below) whereas here we also compute numerically the ion mass fraction distribution 
during the evaporation process so as to take into account the spatial variations of the ion mass fraction within the solution during the evaporation process.

A second objective, which was not addressed in the abovementioned papers, is to discuss the crystal growth by considering the balance between the precipitation kinetics and the convectivediffusive transport processes. This allows us to distinguish two main phases: a very fast one controlled partly by the precipitation kinetics followed by a much longer one controlled by the ion transport. This provides interesting indications for the modeling of the crystal growth process in more complex situations, such as in porous media.

The paper is organized as follows. The experimental set-up is described in Section 2. Experimental results are presented and discussed in Section 3 together with some numerical simulation results. A conclusion is presented in Section 4. The numerical model is presented in the Appendix A.

\section{Experiments}

Evaporation experiments are performed using a circular capillary tube. A circular tube is preferred to a tube of polygonal crosssection so as to avoid complications associated with the development of capillary films along the corners of the tube, i.e. [17,2]. The tubes are borosilicate glass tubes of $1 \mathrm{~mm}$ interior diameter (Hilgenberg ${ }^{\mathcal{O}}$ ). This diameter is denoted by $d$ in what follows. The tube is held either in vertical or horizontal position. The tubes are soaked in an ultrasound bath with detergent solution before use. Then, they are rinsed in deionized water and ethanol before passing in an $\mathrm{O}_{2}$ plasma oven. The sodium chloride solution is prepared with a mass fraction of salt equal to $25 \%$ ( $25 \mathrm{~g}$ of salt for $100 \mathrm{~g}$ of solution), close to the saturation (solubility) value (26.4\%). Salt is provided by Sigma-Aldrich ${ }^{\odot}$, with a purity ensured to be higher than $99.5 \%$. It is diluted in deionised water prepared by using an activated carbon filter and 3 deionizer filters. In order to avoid wetting the top of the tube, the tube is filled from the bottom as sketched in Fig. 1a. As can be seen from Fig. 1, the tube is filled in such a way that a volume of solution is confined between two menisci. Because the top of the tube is surrounded by dry air and the bottom one is in contact with a gas plug saturated in water vapor, evaporation takes place only at the upper meniscus. To form the liquid and gas plugs visible in Fig. 1, the syringe is first connected to a flexible tube. A volume of solution is sucked into the flexible PTFE tube, then air is sucked, then again a volume of

a

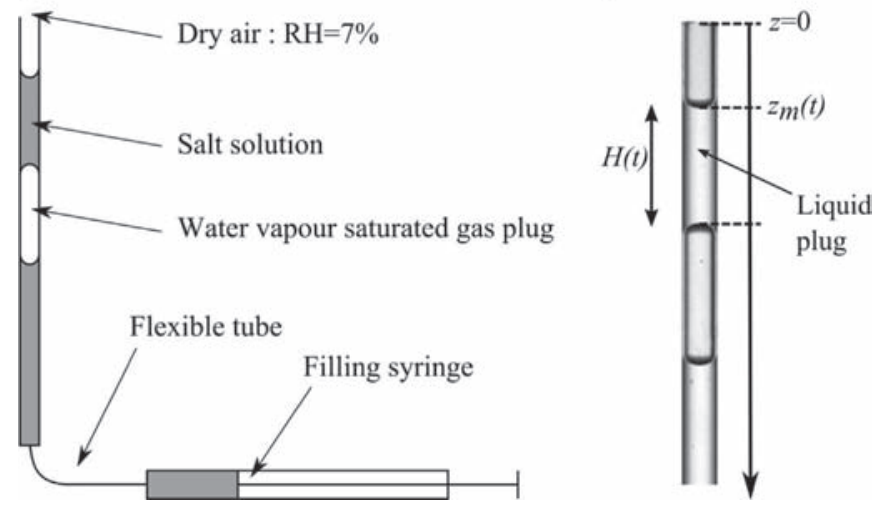

Fig. 1. (a) Schematic of experimental setup, (b) image of the tube obtained by ombroscopy. Liquid and gas phases are in light gray and walls and interfaces appear in dark. The liquid plug of solution is in contact with a dry atmosphere at its top and with a saturated atmosphere at its bottom. The volume of solution at the bottom is connected to the filling syringe. solution. The flexible tube is then glued to the entrance of capillary tube and the adjacent liquid and gas plugs are slowly injected into the capillary tube to obtain the phase distribution shown in Fig. 1b.

The tube is set in an enclosure at controlled temperature ( $T$ $\sim 22{ }^{\circ} \mathrm{C}$ ) and relative humidity ( $\mathrm{RH} \sim 7 \%$ ) thanks to a lithium bromide ( $\mathrm{LiBr}$ ) solution covering the bottom of the enclosure. The temperature and relative humidity in the enclosure are measured and recorded during the experiment. All the data are obtained from successive images of the capillary tube during an evaporation experiment. Images are taken with a Nikon ${ }^{\circledR}$ D70 camera with an AFS micro Nikkor $105 \mathrm{~mm}$ lens for the low acquisition frequency (typically $0.0033 \mathrm{~Hz}$ ), and a film camera CCD Sensicam $\mathrm{PCO}^{\odot}$ for higher frequency (typically $0.5 \mathrm{~Hz}$ ). These devices are interfaced to a computer thanks to Camera Control Pro $2^{\odot}$ and Camware ${ }^{\odot}$ software, respectively. The objective is to follow the receding meniscus during evaporation in order to: i) measure its kinetics, ii) determine the size of liquid plug, which gives access to values of saturation. Also the visualization enables us to measure the crystallization kinetics. As can be seen from Fig. 1, gas and liquid phases appear in light gray and wall and interface in dark with the ombroscopy method used for this experiment.

Results are presented for seven experiments (Table 1), four with the tubes held in a vertical position and three with the tube in a horizontal position. This is basically the same experiment, which is repeated seven times. The differences lie in the position of the tube, vertical or horizontal, the initial position $z_{m 0}$ of the receding meniscus within the tube and in the initial volumes of gas and liquid plugs.

Looking at Fig. 1b, one may wonder whether the dark thick lines along the vertical edges of the tube image in the regions of the tube occupied by the gas phase corresponds to liquid films along the tube inner wall. These dark lines are, however, due to an optical effect associated with the curvature of the tube wall. They are also observed in a perfectly dry tube. Note that the contact angle of a salt solution on glass is on the order of $40-45^{\circ}$ [18]. Thus we are far from a situation of perfect wetting. A very thin film, of a few nanometers, might be present. However, we do not believe that such a film, if any, can play a role in the investigated problem.

\section{Results and discussion}

\subsection{Evaporation kinetics}

A first step is to control that the evaporation kinetics before the onset of crystallization is in good agreement with the available

Table 1

Global and local supersaturation obtained for each experiment (see Section 3.4). The global supersaturation is obtained from an average mass balance whereas the local supersaturation is obtained from determining numerically the ion mass fraction distribution within the tube; Meaning of letters is: V: vertical, $\mathrm{H}$ : horizontal, T: top, B: bottom, M: middle; $z_{m 0}$ is the initial position of the receding meniscus measured from tube entrance, $H_{0}$ is the initial length of liquid plug; $D_{s}$ is the molecular diffusion coefficient of the ions in the liquid phase $\left(D_{s}=1.310^{-9} \mathrm{~m}^{2}\right)$ s). The Peclet number $P e$ is defined and discussed in $\S 3.2$. Note that the images in Fig. $1 \mathrm{~b}$ and Fig. 3 do not correspond to one of the experiments mentioned in the table. They correspond to an experiment performed with a shorter initial liquid plug designed to visualize the fall of the crystal within the plug (see text).

\begin{tabular}{llllllll}
\hline Experiment \# & 1 & 2 & 3 & 4 & 5 & 6 & 7 \\
\hline Tube orientation & $\mathrm{V}$ & $\mathrm{V}$ & $\mathrm{V}$ & $\mathrm{V}$ & $\mathrm{H}$ & $\mathrm{H}$ & $\mathrm{H}$ \\
$z_{m 0}(\mathrm{~mm})$ & 1.4 & 3.7 & 1.3 & 1.9 & 3.5 & 8.8 & 4.0 \\
$H_{0}(\mathrm{~mm})$ & 27.3 & 19.4 & 19.4 & 16.9 & 18.6 & 18.2 & 20.5 \\
Crystal growth site position & $\mathrm{T}$ & $\mathrm{B}$ & $\mathrm{B}$ & $\mathrm{B}$ & $\mathrm{T}$ & $\mathrm{M}$ & $\mathrm{T}$ \\
Initial Peclet number $P e_{0}=\frac{\mathrm{d} z_{m}(0) H(0)}{\mathrm{dt}}$ & 3.73 & 1 & 2.86 & 1.7 & 1.01 & 0.4 & 0.98 \\
Global supersaturation & 1.35 & 1.34 & 1.46 & 1.32 & 1.22 & 1.40 & 1.62 \\
Local supersaturation & 1.59 & 1.53 & 1.62 & 1.52 & 1.41 & 1.51 & 1.78 \\
& & & & & & & \\
\hline
\end{tabular}


theoretical prediction. The problem of evaporation in a circular tube is classically referred to as the Stefan diffusion tube problem, after Stefan (1871), who was the first to study it. The solution to this problem, which is described in text books, e.g. [19], reads:

$z_{m}(t)=\sqrt{2 M \frac{{ }_{v} P D_{v}}{\rho_{\ell} R T} \ln \left(\frac{1-y_{v \infty}}{1-y_{v e}}\right)\left(t-t_{0}\right)+z_{m}^{2}\left(t_{0}\right)}$,

where $z_{m}$ is the position of receding meniscus in the tube measured from tube entrance; $M_{v}$ is the water molecular weight, $P$ and $T$ are the gas total (atmospheric) pressure and temperature in enclosure, $\rho_{\ell}$ is the liquid density, $R$ is the ideal gas constant, $D_{v}$ is the molecular diffusion coefficient of water vapor in the gas phase (considered as a binary mixture of air and water vapor), $y_{v \infty}$ is the water vapor molar fraction in the surrounding air and $y_{v e}$ is the equilibrium molar fraction of vapor at the meniscus. Index 0 refers to the initial time. Eq. (2) is referred to as "Stefan's law" in what follows.

As mentioned in the Section 1, we developed a numerical solution to determine the ion mass fraction distribution within the tube. This numerical approach is described in the Appendix A. Before considering the computation of ion mass fraction within the plug during the evaporation process, a first step is to verify that the numerical solution leads to a correct prediction of the receding meniscus dynamics induced by the evaporation process. To this aim, the case of evaporation of pure water was first simulated and the solution compared to the theoretical prediction given by Eq. (2). The comparison is shown in Fig. 2 and corresponds to the curves labeled "Simulation test". As can be seen, a good agreement is found between the numerical solution and the prediction given by Eq. (2). Thus, the receding meniscus dynamics is correctly computed for pure water. The next step is to take into account the presence of the ions in the plug.

Eq. (2) cannot be used anymore as reference solution since this equation is obtained based on the assumption that the equilibrium molar fraction of vapor at the meniscus is constant. This is a correct assumption for pure water under negligible temperature variation condition, which is the case in our experiment. However, the presence of ions modifies the water activity $a_{w}=P_{v e}(C) / P_{v e}(0)$, which is the ratio of equilibrium vapor pressure at the surface of a sodium chloride aqueous solution (of ion mass fraction $C$ ) to the equilibrium vapor pressure at the surface of pure liquid water. As discussed in [5], the water activity decreases with increasing ion mass fraction $C$. In our experiment, the ion mass fraction increases progressively. This affects the water vapor partial pressure at the surface of receding meniscus and thus the evaporation kinetics.

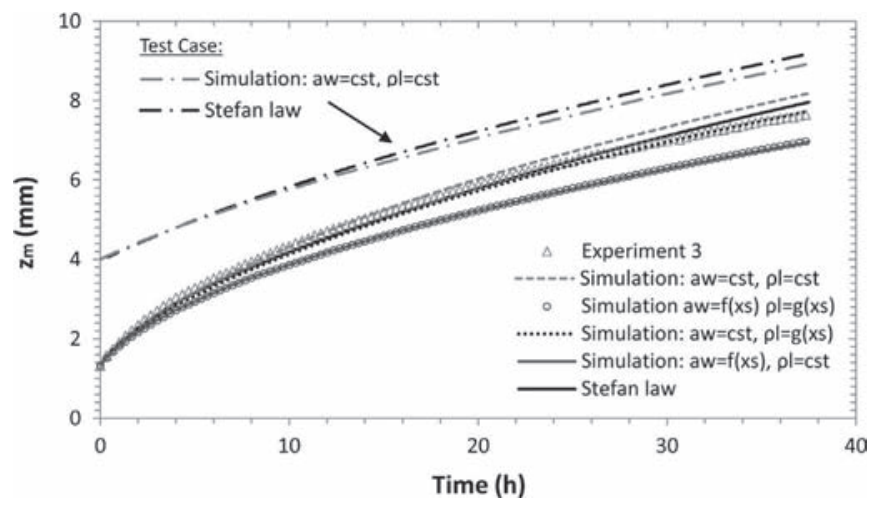

Fig. 2. Position of receding meniscus versus time. Experimental results are compared with Stefan's law calculated with fluid properties corresponding to the initial state, and numerical simulations considering various options as regards the variations of water activity and solution density as a function of ion mass fraction. Note that the simulation curves corresponding to $a_{w}=\operatorname{cst}, \rho_{\ell}=g\left(x_{s}\right)$ and $a_{w}=f\left(x_{s}\right)$, $\rho_{\ell}=$ cst lie on the same line and cannot be discerned from each other.
This effect was taken into account in the numerical solution of the problem summarized in the Appendix A. We have also taken into account the variation of solution density with ion concentration in the numerical solution but not the variation of salt diffusion coefficient with ion concentration because the variation is weak according to the data reported in [20]. One problem is that the variations of density and water activity are only known in the range of ion mass fraction $\left[0-x_{\text {sat }}\right]$ whereas the ion mass fraction can reach values above the solubility mass fraction in the presence of supersaturation. We have tested two options: the first one is to assume that the density (and activity) cannot be greater (lower respectively) that the solubility values, i.e. $\rho_{\ell}\left(x_{s}\right)=\rho_{\ell}\left(x_{s a t}\right)$ and $a_{w}\left(x_{s}\right)=a_{w}\left(x_{s a t}\right)$ for $x_{s} \geq x_{\text {sat }}$. The second option consists in taking into account the variations of activity and density above the solubility, that is in the range $\left[x_{s a t}-x_{c}\right]$, where $x_{c}$ is the ion mass fraction corresponding to supersaturation. The density in the range is determined using the same expression as for lower salt mass fraction, namely, $\rho_{\ell}\left(x_{s}\right)=\frac{\rho_{\ell}(0)}{1-0.7 x_{s}}$, whereas the variation of activity over the full range $\left[0, x_{c}\right]$ can be obtained from the data reported in [21].

The comparisons between the experimental data corresponding to experiments \#3 in Table 1, the prediction given by Eq. (2), i. e. Stefan's law neglecting the variation in the equilibrium vapor partial pressure and the density, and the numerical solutions, thus taking into account the decrease in the equilibrium vapor partial pressure and the increase in the density with an increasing ion mass fraction, are presented in Fig. 2. Similar comparisons were obtained with the other experiments.

As can be seen, the meniscus displacement is slower with the solution considering the variation of properties compared to a solution with constant properties. This was expected since the density increases with an increasing ion mass fraction while the water activity decreases. However, the best agreement with the experimental data is obtained assuming $a_{w}\left(x_{s}\right)=a_{w}\left(x_{s a t}\right)$ for $x_{s} \geq x_{\text {sat }}$ and $\rho_{\ell}\left(x_{s}\right)$ varying above $x_{\text {sat }}$. There is clear discrepancy with the experimental results when values $a_{w}\left(x_{s}\right)$ vary in the range $\left[x_{\text {sat }}-x_{c}\right]$. However, it can be noted that this is not in agreement with the experimental results reported in [21] or [14], which indicate that the water activity decreases below $a_{w}\left(x_{s a t}\right)$ when $x_{s} \geq x_{\text {sat. }}$. In fact, the liquid plug is not perfectly stable during the experiments, presumably due to some vibrations in the experiment room. The associated uncertainty might explain the discrepancy.

Also, it may appear surprising that the simulations for $a_{w}=f\left(x_{s}\right)$, $\rho_{\ell}=g\left(x_{s}\right)$ and $a_{w}=f\left(x_{s}\right), \rho_{\ell}=$ cst lead to almost the same result in Fig. 2 whereas the consideration of a variable density has clearly an impact when $a_{w}=$ cst. Actually, the variation of activity in our simulation is expressed as a function of ion mass fraction, which is the ratio of ion concentration to solution density. The result is that the variation of $x_{s}$ is different in both simulations and this explains the observed result.

To close this section, it can be also noted that there should exist a vapor flux in the air gap between the top (evaporating) plug and the lower (saturated) plug since the activity becomes different between the two menisci closing this gap owing to the progressive change in the ion concentration in the upper plug. Estimate of this flux, which is directed from the lower plug to the upper plug, shows that it can be neglected in the analysis.

\subsection{Location of first precipitation spot}

As the meniscus recedes into the tube, the ion concentration increases in the shrinking liquid plug until first crystals become visible and grow. Only one crystal growth site was observed in the experiments summarized in Table 1 . As reported in Table 1 , there are three main places in the tube where this crystal growth is observed. Generally, crystal growth takes place at a meniscus. The 
growth site can be located either at the inner (liquid) surface of the receding meniscus (position denoted by letter " $t$ " in Table 1 ), at the liquid side of bottom meniscus (letter "b" in Table 1, which refers in fact to the immobile meniscus located at the other end of liquid plug and is indeed the bottom meniscus when the tube is vertical). In one experiment (experiment \#6), however, the crystal growth took place in the middle of tube. The growth site was usually located near the receding meniscus in the experiments with the tube in the horizontal position (with the exception of experiment \#6 where growth took place in the middle of the tube). The situation seems at first sight different when the tube is held in the vertical position, where the most likely growth site was the bottom (immobile) meniscus. However, the observation of series of images taken with a bigger zoom and at a higher frequency $(0.5 \mathrm{~Hz})$ actually in a dedicated experiment showed that the crystals first form at the upper meniscus also when the tube is in vertical position. Then the crystal, of density greater than the solution density, fell into the liquid plug until it reached the bottom meniscus, where most of its growth took place. This is illustrated in Fig. 3. The conclusion is therefore that the most likely place of crystal formation is the receding meniscus. However, as can be also seen from Fig. 3, the crystal might stay attached to the upper meniscus where the interfacial forces are sufficient to overcome the gravity forces acting on the crystal. Note also the formation of a third growth site in Fig. 3 in the upper part of the receding meniscus on the left, leading to salt creeping along the inner wall of the tube. In the particular experiment shown in Fig. 3, three growth sites can be seen. As mentioned before, only one crystal growth site was observed in the experiments summarized in Table 1.

The observation of the growth site in the middle of the plug in experiment \#6 can be related to the lower initial Peclet number $P e_{0}$ characterizing this particular experiment. This is due to the initial receding meniscus position deeper inside the tube compared with the other experiments. As discussed in more details in §3.4, a lower Peclet number means a more uniform ion mass fraction distribution within the liquid plug and therefore a lower probability to observe the precipitation at the receding meniscus.

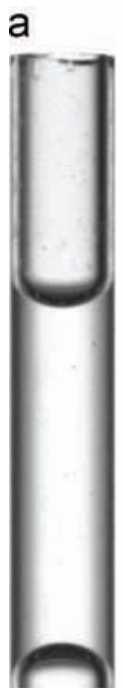

b

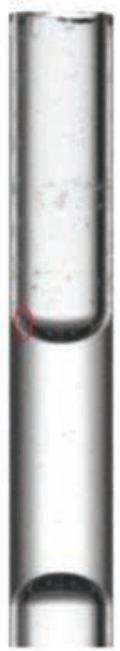

$t_{n}$

h
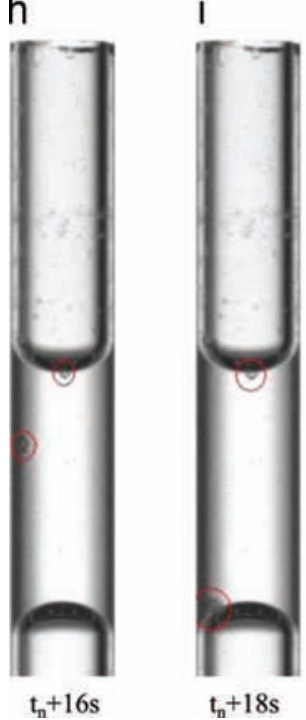

C

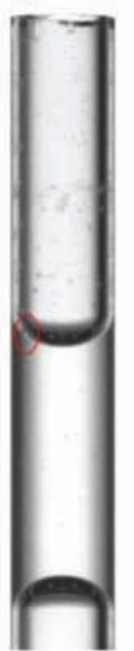

$t_{n}+2 s$

j

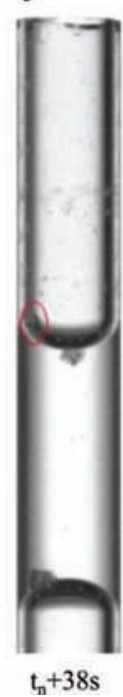

d

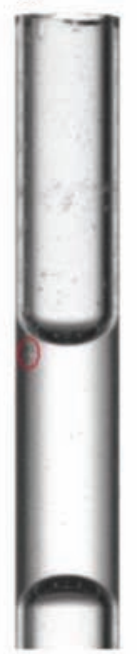

$t_{n}+6 s$

$\mathrm{k}$ e

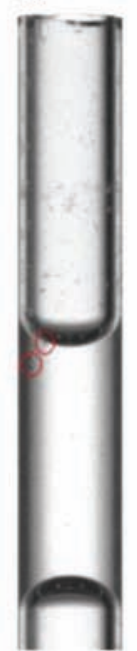

$t_{n}+8 s$

I

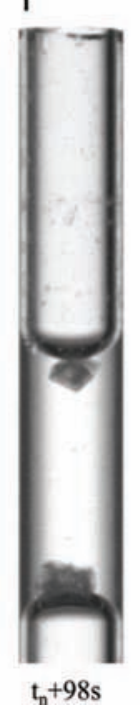

f

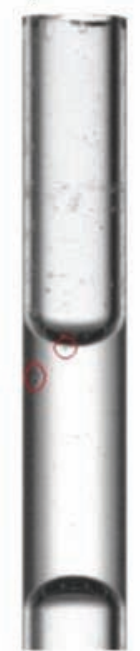

$t_{n}+10 s$

m

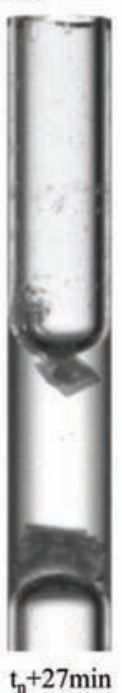

g

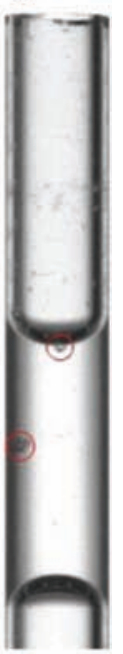

$t_{n}+14 s$

n

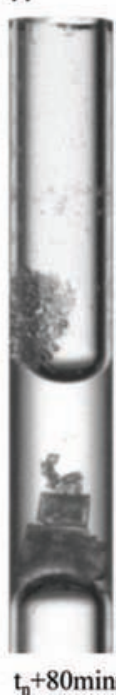

Fig. 3. Series of pictures obtained by ombroscopy showing the sodium chloride crystal growth in the tube. Red circles locate crystals. Time is counted from first precipitation time $t_{n}$, which is the time when the first crystal is detected by visual inspection of the image (For interpretation of the references to color in this figure legend, the reader is referred to the web version of this article.). 
This is also consistent with the lower discrepancy between the local and global supersaturations for experiment \#6 compared with the other experiments.

The fact that the most likely place of crystallization is at the receding meniscus can be explained from the numerical computation of the ion mass fraction field within the plug during the evaporation process. The method of solution is described in the Appendix A. Owing to the discrepancy observed between the experiment and the simulation as regards the receding meniscus dynamics when we consider the conditions that a priori must apply (see Section 3.1), we have decided to perform the computation of this field in such a way that the dynamics of receding meniscus is in almost perfect agreement with the experimental dynamics. Nevertheless, we have also checked the other options corresponding to the various curves depicted in Fig. 2. We found that the results were not significantly sensitive to the option considered provided that the solution density variation was taken into account in the simulation. Neglecting the density variation leads to overestimation of the ion concentration at the receding meniscus compared to the simulations taking into account the density variation.

Fig. 4 shows a representative example of the computed ion mass fraction field within the liquid plug. As can be seen, the simulations indicate the formation of dissolved salt concentration gradients during the evaporation process. The ion mass fraction is greater in the region adjacent to the receding meniscus. The ion mass fraction is the greatest near the contact line, i.e. the line where solid, liquid and gas meets. This is fully consistent with the observations since first crystals are generally seen in the wedge formed by the meniscus and the tube wall near the contact line.

Physically, the distribution depicted in Fig. 4 can be explained as follows. When the receding meniscus moves over a distance $d z$ during the time $d t$, this corresponds to the evaporation of a certain volume $V$ of solution. It is easy to show that this volume is actually equal to $d z \pi d^{2} / 4$ independently of the shape of the meniscus (provided that this shape does not change over the time $d t$ ). The

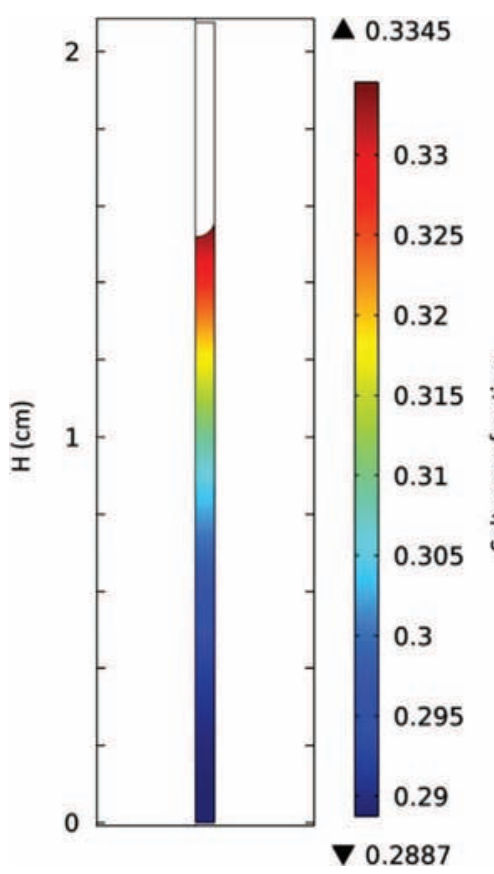

Fig. 4. Example of computed salt mass fraction distribution within the liquid plug after $21 \mathrm{~h}$ of evaporation (simulation of experiment \#3). Note that only a half vertical section of the tube is shown because of the symmetry of the problem. (For interpretation of the references to color in this figure legend, the reader is referred to the web version of this article.) mass of salt contained in this volume is $m_{s}=x_{s} \rho_{\ell} V$. Since the ions do not evaporate, this is the mass of salt added to the plug as the results of the displacement of the meniscus over the distance $d z$. This mass is added locally along the meniscus inner boundary. When the motion of the meniscus is too fast, diffusion cannot homogenize ion mass fraction within the plug and the salt concentration increases near the receding meniscus as illustrated in Fig. 4. This leads to introduce a dimensionless number for characterizing the competition between the two effects, namely the ion diffusion and the fact that ions are added with a certain rate along the receding meniscus, expressed as:

$\frac{d m / d t}{J_{s}}=\frac{\text { ion production rate at receding meniscus }}{\text { diffusion rate }}$.

The diffusion rate is given by: $J_{S} \approx \rho_{\ell} \frac{\pi d^{2}}{4} D_{s} \frac{X_{S}}{H}$, leading to:

$P e=\frac{\left(d z_{m} / d t\right) H}{D_{s}}$,

which has the form of a Péclet number and is therefore denoted by $\mathrm{Pe}$. Accordingly, it is expected that the ion mass fraction is uniform within the plug when $P e$ " 1 and not uniform with greater ion mass fractions near the receding contact line when $\mathrm{Pe} \approx \mathrm{O}(1)$ or greater.

Then the greater concentration near the contact can be qualitatively explained as follows when $P e$ is greater than one. Suppose that an excess ion mass fraction is distributed all along the meniscus. Then it is clear that diffusion will be more efficient near the middle of the receding meniscus (the point of the meniscus which is the most advanced within the plug), which is closer to the less concentrated solution occupying the plug than the points of the meniscus located closer to the contact line. In brief, the confinement effect within the wedge formed by the meniscus and the tube wall near the contact line explains the greater ion mass fraction in this region. Note that the excess mass fraction is therefore uniform along a moving flat meniscus.

From the above consideration, it is possible to determine when the ion mass fraction can be reasonably expected to be nearly uniform within the plug. This might help experimentalists to set up experiments so as to have a nearly uniform ion mass fraction when crystallization occurs, without using numerical simulations. From Eq. (2), we get:

$\frac{d z_{m}}{d t}=\frac{A}{z_{m}}$,

where $A=M \frac{v}{\rho_{\ell} R D_{v}} \ln \left(\frac{1-y_{v \infty}}{1-y_{v e}}\right)$. Combining Eq. (4) and Eq. (5) leads to: $P e=\frac{A H}{z_{m} D_{s}}$,

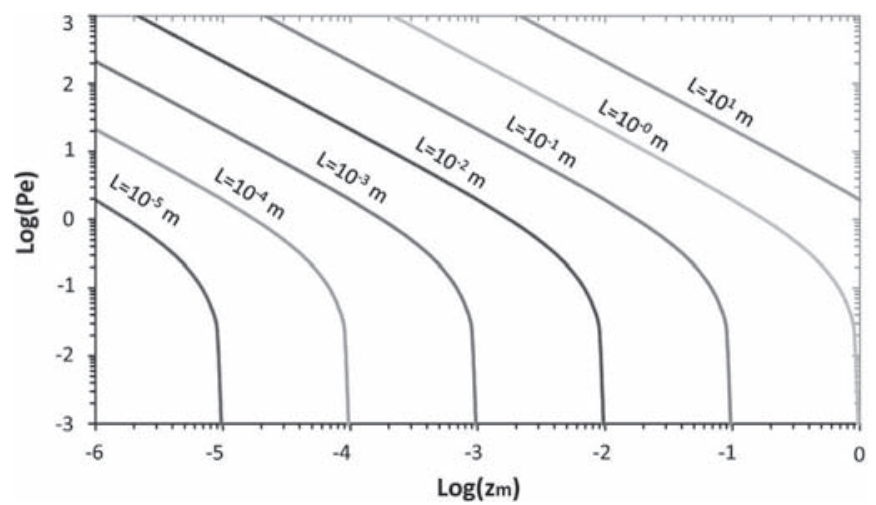

Fig. 5. Pe number as a function of receding meniscus position within the tube. $L$ is the length of computational domain, which corresponds to the section of the tube containing the upper plug; $L=H(t)+z_{m}(t)$ (see Fig. 1b). 
As can be seen in Fig. 5 (for the case $y_{v \infty}=0$ ), the hypothesis of a homogeneous salt concentration can be often not relevant. For example, when the meniscus is located at $1 \mathrm{~mm}$ from the entrance of a $3 \mathrm{~cm}$ long tube, Pe number is equal to 6.23 , much higher than 1.

\subsection{Convective motion along the receding meniscus}

It is well known that the structure of liquid velocity field in the vicinity of an evaporating meniscus can be complex with possible rotating vortices in the region adjacent to the meniscus, e.g. [22]. The convective rolls reported in [22] were induced by the temperature variations along the meniscus generated by the preferential cooling near the contact line, which is the place of the highest evaporation flux. However, this Marangoni flow is expected to be insignificant in our experiments owing to the quite low evaporation rates and the operating fluid used. Nevertheless, the evaporation flux is not uniform along the meniscus with greater evaporation fluxes near the contact line as illustrated in Fig. 6. According to our numerical approach (see Appendix A), which thus assumes negligible temperature variations, the evaporation flux variation along the meniscus induces a double convective loop in the liquid as shown in Fig. 6. It could be concluded that this convective flow contributes to the transport of ions from the middle of meniscus to the contact line region. However, the simulations indicate that the maximum velocity $V_{\ell \text { max }}$ in this flow pattern is at most on the order of $10^{-8} \mathrm{~m} / \mathrm{s}$. If we define a local Péclet number based on this convective velocity and the tube diameter:

$P e_{m}=\frac{V_{\ell \max } d}{D_{s}}$,

a value of about $10^{-2}$ is obtained. The conclusion is therefore that the ion concentration field is in fact almost not affected by the convective motion depicted in Fig. 6. The greatest concentration near the contact line is not due to this convective effect but to the confinement effect briefly discussed in the previous section.

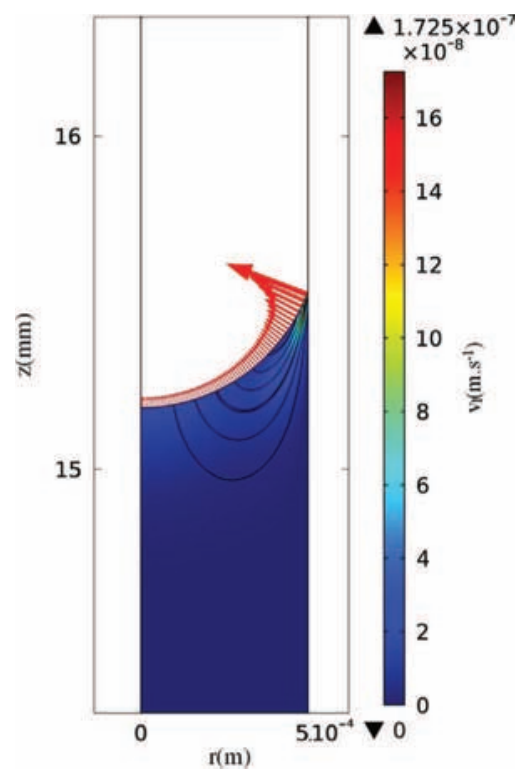

Fig. 6. Computed flow pattern (stream lines) induced in the liquid in the region under the receding meniscus. The red arrows indicate the distribution of evaporation flux along the meniscus (For interpretation of the references to color in this figure legend, the reader is referred to the web version of this article.).

\subsection{Supersaturation}

A first estimate of supersaturation can be obtained from a simple mass balance. Knowing the initial volume $V_{0}$ of the liquid plug, the initial ion mass fraction in the plug $x_{0}$, the average ion mass fraction $\bar{x}_{c}$ when first crystals are detected in the images is then given by:

$\frac{\bar{x}_{c r}}{x_{0}}=\frac{\rho_{\ell}\left(x_{0}\right) V_{0}}{\rho_{\ell}\left(\bar{x}_{c r}\right) V_{c r}}$,

where $V_{c r}$ is the volume of the liquid plug right before first crystals are visible; $\rho_{\ell}$ is the density of the solution, which depends on the ion mass fraction, $\rho_{\ell}=\frac{\rho_{e}}{1-0.7 x_{s}}$. Combining the latter with Eq. (3) leads to:

$\frac{\bar{x}_{c r}}{x_{0}}=\frac{1}{\omega+0.7 x_{0}(1-\omega)}$,

where $\omega=V_{c r} / V_{0}$. Expressing the supersaturation, Eq. (1), in terms of ion fraction leads to:

$S=\frac{\bar{x}_{c r}}{M_{S}\left(1-\bar{x}_{c r}\right) m o_{s a t}}$,

which can be easily computed from the determination of $\bar{x}_{c}$ using Eq. (9). This leads to the values referred to as the global supersaturation values in Table 1.

Averaging over the seven experiments yields $S=1.39 \pm 0.1$, which is slightly lower than the values $(\sim 1.6)$ reported in [14] but still greater than one. This lower value compared with the results of [14] is explained by the fact that the ion mass fraction is not uniform within the liquid plug in our experiments. Therefore, the numerical simulations were used to determine the local ion mass fraction where and when the first precipitation spot was observed in each experiment. Using these values in Eqs. (9) and (10) led to the values referred to as the local supersaturation in Table 1 . As can be seen the local values are greater than the global values with a lower relative variability. Averaging over the seven experiments, one obtains $S=1.57 \pm 0.1$, which is very close to the values reported in [14]. The global method was used in [14] for determining the supersaturation. Their experimental conditions were different than ours. Using values deduced from their article in Eq. (6) leads to $P e<0.05$ for their experiments. This is much smaller that the values of Peclet number in our experiments (see Table 1). This low value of Peclet number consistently explains why the consideration of the average ion concentration was sufficient in their case.

\subsection{Crystal growth kinetics}

First insights on the crystal growth kinetics can be gained from the spatiotemporal diagram shown in Fig. 7, for the case here of the tube in vertical position. This diagram shows the receding of the upper meniscus as a function of time and the growth of the crystal attached to the immobile bottom meniscus. This diagram is obtained by plotting the one pixel wide line located in the middle of each image. This line is schematically shown in Fig. 7. It corresponds to the vertical dashed line on the right in the figure.

In the experiment shown in Fig. 7, there is only a single crystal growing. This is the situation generally observed in our experiments. As can be seen, one can distinguish three main phases. During the first phase, when no crystal is present, the ion mass fraction increases in the liquid as a result of evaporation. Then there is the short phase in which the crystal appears right under the receding meniscus and falls within the plug until it reaches the bottom meniscus. This short phase is not visible in Fig. 7 and is therefore not considered as a distinct phase in this section. The second phase corresponds to rapid crystal growth indicated by a 
quasi-vertical line in spatiotemporal diagram in Fig. 7. During the third phase the growth becomes much slower.

Regarding the crystal growth kinetics, one can therefore distinguish two main phases: a first phase where the growth is quite fast and a second phase where the growth is much slower. This can be further illustrated from the computation of the crystal growth velocity $w_{c r}$, which is defined by:

$w_{c r}=\frac{d \ell}{d t}$,

where $\ell$ is the height of crystal as sketched in Fig. 7. The crystal growth velocity $w_{c r}$ is computed from the series of images of crystal developing in the tube (again it is computed from the one pixel wide middle line used to plot the spatiotemporal diagram). This led to the evolution of crystal growth velocity depicted in Fig. 8, which clearly shows the short phase characterized by a much higher growth velocity from the second phase where the growth velocity is much lower.

As can be seen from Fig. 8, the duration of the first phase is quite short, on the order of $20 \mathrm{~min}$. Also, Fig. 8 shows that the numerical simulation based on the model summarized in the Appendix A leads a qualitative agreement with the experiment. The two phases are well reproduced and the duration of the first phase, although shorter than in the experiment, is on the same

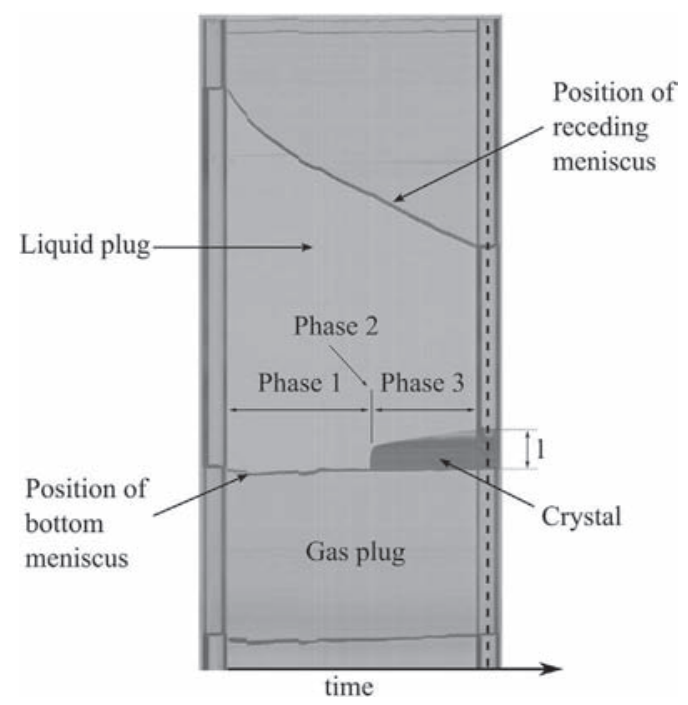

Fig. 7. Spatiotemporal diagram. On the left: initial time picture, on the right: final time picture, in between: meniscus position versus time, each pixel column corresponds to a picture section in the middle of the tube. A crystal (dark region) appears after $37 \mathrm{~h}$ of evaporation. The diagram shown here corresponds to experiment \#2.

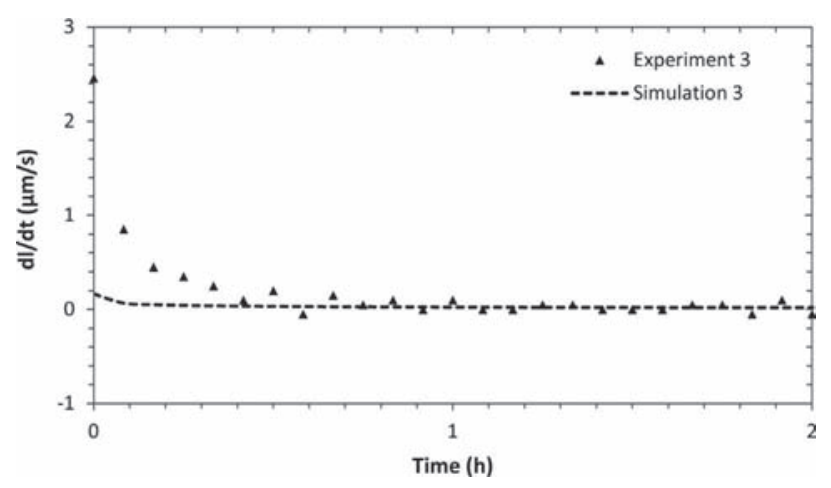

Fig. 8. Crystal growth velocity versus time; $t=0$ corresponds to the time when crystal becomes visible for the first time in the images. order as in the experiment. The shorter duration of the first phase in the simulation is attributed to the consideration of a crystal shape much simpler than the shape seen in the experiment. As indicated in the Appendix A, the crystal was actually assumed to fully occupy the tube cross-section and the crystal-liquid interface was assumed to be perfectly flat.

To discuss further these results, we recall the expression of the precipitation kinetics proposed by [23]:

$\frac{d \ell}{d t}=K_{c r}(S-1)^{g_{c r}}$

where $K_{c r}$ is the growth parameter, depending on temperature and $g_{c r}$ is the crystal growth exponent. The growth parameter $K_{c r}$ can be expressed thanks to the Arrhenius law: $K_{c r}=C_{c r} \exp \left(-E_{a} / R T\right)$ where $C_{c r}$ is a constant, $E_{a}$ the activation energy and $T$ the temperature. Summarizing different values of the literature, [24] suggests the following values for each coefficient: $C_{c r}=1.1410^{4} \mathrm{~m} /$ $\mathrm{s}, E_{a}=58,180 \mathrm{~J} / \mathrm{mole}$ and $g_{c r}=1$ for $\mathrm{NaCl}$. Eq. (12) indicates that the growth is directly linked to the existence of ion in excess (the supersaturation $S$ should be greater than 1 ).

The fact that there are two well distinct phases in the crystal growth kinetics can then be discussed first from the consideration of the Damkhöler number $D a$, which compares the reaction time (precipitation time) ant the transport (convection+diffusion) time scale. The Damkhöler number Da can be expressed here as:

$D a=\frac{K_{c r} H}{D_{s}}$.

At the beginning of cristal growth, $D a \approx 6$. We recall that $D a$ $>1$ indicates that the reaction is fast compared to transport. This means that the crystal consumes all the dissolved salt in excess near it surface when crystallization starts, and then the process is controlled by transport. This value of Damkhöler crystal thus suggests that the growth is first limited by the reaction and the transport over a short first phase until the solubility concentration is reached at the crystal surface. In the second period, much longer, precipitation is limited by transport only.

This scenario is confirmed from the numerical computation of the ion mass fraction field within the liquid plug during the evaporation - crystal growth process. Again, the mathematical model and the method of solution are described in the Appendix A. The results of simulation are depicted in Figs. 9 and 10 for the case of experiment \#3. Similar results were obtained for the other experiments. $z=0$ corresponds to the crystal-liquid interface in Fig. 9 whereas $z=z_{\max }$ corresponds to the receding meniscus position $\left(z_{\max }\right.$ is the point where the curves stop on the right in Fig. 9). As can be seen from Fig. 9, the phase of rapid growth corresponds to a short phase where the supersaturation

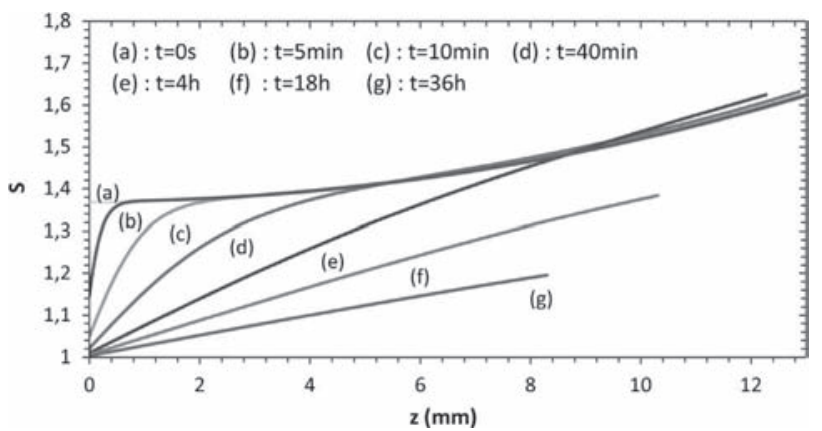

Fig. 9. Computed variation of supersaturation in the salt solution in the liquid plug at different times in the configuration of experiment $\# 3 ; z=0$ corresponds to the crystal interface and the greatest value of $z$ for each curve corresponds to the liquid gas interface, i.e. the receding meniscus. 


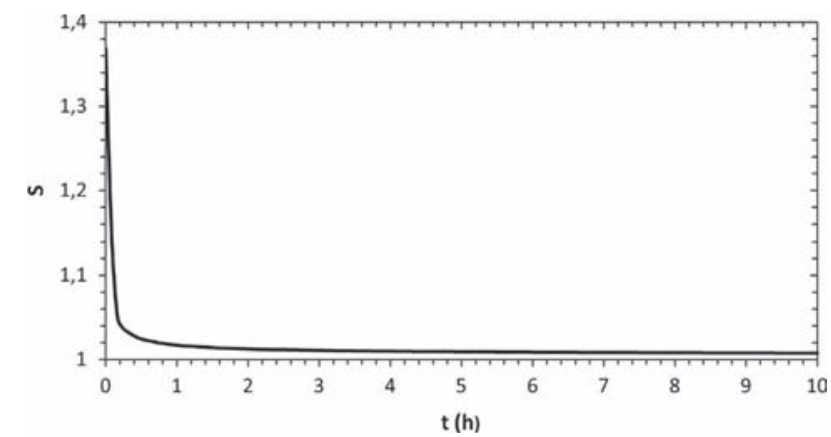

Fig. 10. Computed variation of supersaturation at crystal surface $(z=0)$ in the configuration of experiment \#3.

is greater than 1 at the crystal surface in contact with the liquid (see the decrease in supersaturation at $z=0$ ). This is still more visible in Fig. 10 which shows the variation of supersaturation right at the crystal surface. This is a phase where the crystal growth is controlled by both the precipitation kinetics and transport (since the supersaturation decreases at $z=0$ ). As shown in Fig. 9, this phase is characterized by a strong ion mass fraction gradient at the crystal surface, especially at the very beginning of the growth. In this phase, the crystal clearly consumes more salt than the amount of salt in excess produced as a result of evaporation. In other terms, the average ion mass fraction in the plug markedly decreases during this phase. This phase lasts about $15 \mathrm{~min}$. Then the ion mass fraction becomes very close to the solubility at $z=0$ (which corresponds to $S \sim 1$ ) and the growth is then controlled by the transport only. This corresponds to the phase of slow crystal growth. As depicted in Fig. 9, the ion concentration gradient at the crystal surface progressively decreases during this phase and the ion mass fraction tends to become more uniform over the liquid plug. This phase, which is controlled by the transport and the volume variation of the liquid plug, could be computed imposing $x_{s}=x_{\text {sat }}$ at the crystal-liquid interface and solving only the transport equations without considering the precipitation kinetics Eq. (12).

We have seen in $\S 3.3$ that ion mass gradients can be induced by the motion of the receding meniscus (when Pe is sufficiently high). One can also wonder whether the motion of crystal-liquid interface can induce convective effects in the liquid and therefore also gradients in the ion mass fraction within the liquid plug. Similarly as in $\S 3.3$, a first insight into this question can be gained from the consideration of a Peclet number $P e_{c r}$ defined by:

$P e_{c r}=\frac{(d \ell / d t) H}{D_{s}}$

where $d \ell / d t$ is a characteristic velocity of the flow induced in the liquid plug by the crystal growth. Using experimental values of $d \ell / d t$ during the phase of fast crystal growth leads to $P e_{c r} \sim 17$, which clearly suggests that the crystal growth induces ion mass fraction convective effects in the plug and thus ion mass fraction gradients, at least during the phase of rapid growth.

Another interesting result drawn from simulations concerns the direction of average velocity within the plug induced by the crystal growth. One could be tempted to predict that this velocity near the crystal is directed toward the receding meniscus according to the naive view that the crystal is pushing the liquid. Actually, the crystal growth induced a flow in the opposite direction, that is directed toward the crystal and not away from the crystal. Note, this holds however only under condition of negligible salt solution density gradient. This can be understood from the expression of liquid velocity at crystal surface that can be deduced from Eq. (A15) in the Appendix A. The projection of Eq. (A15) along the $z$-direction leads to:

$v_{\ell z}=w_{c r z}\left(1-\frac{\rho_{c r}}{\rho_{\ell}}\right)$,

where $w_{c r z}$ is the crystal-solution interface velocity. Since the crystal density $\rho_{c r}\left(\rho_{c r}=2170 \mathrm{~kg} / \mathrm{m}^{3}\right)$ is greater than the solution density $\rho_{\ell}$, the flow induced in the liquid is in the direction opposite to the crystal growth direction. The convective effect induced in the liquid by the crystal growth thus increases the transport of ions toward the growing crystal. Hence the water is pushed away by the crystal, the ions are directed toward the crystal and the solution, i.e. the mixture of the two species water and dissolved salt, is directed toward the growing crystal interface.

Finally, we note that it is easy to determine the crystal growth rate in the low Peclet limit when the ion mass fraction can be considered as uniform within the plug. Since we know from $§ 3.4$ that the supersaturation is quite significant at the onset of precipitation, a first step is to consider that the fast crystal growth phase corresponds to a phase where all the salt in excess precipitates. The second phase, much slower, would be then controlled by the slight increase in the ion mass fraction due to the shrinking of liquid plug. It is assumed that the resulting increase in the ion mass fraction precipitates immediately. Thus, the system would operate in the second phase under quasiequilibrium condition between the crystal and the solution $\left(x_{s} \sim x_{\text {sat }}\right.$ throughout the solution) in this low Pe regimes. Under these circumstances, the crystal growth rate during the second phase can be predicted from the simple mass balance:

$\rho_{c r} \frac{d \ell}{d t} \approx-\rho_{\ell} x_{s a t} \frac{d H}{d t} \approx \rho_{\ell} x_{s a t}\left(\frac{d z_{m}}{d t}+\frac{d \ell}{d t}\right)$,

where $\rho_{c r}$ is the crystal density $\left(\rho_{c r}=2160 \mathrm{~kg} / \mathrm{m}^{3}\right)$, which gives:

$\frac{d \ell}{d t} \approx \frac{\rho_{\ell} x_{s a t}}{\left(\rho_{c r}-\rho_{\ell} x_{s a t}\right)} \frac{d z_{m}}{d t}$.

Note that we assumed a compact crystal.

The prediction given by Eq. (17) is compared with the full simulations in Fig. 11.

As expected, Eq. (17) leads to a lower bound in the crystal growth velocity. In the case of our experiments, the distribution of ions is far from the quasi-uniform distribution expected in the low Peclet limit and the crystal growth is much faster. This could be also simply anticipated from the computation of the diffusion time $H^{2} / D_{s}$, which is on the order of $40 \mathrm{~h}$ for our experimental conditions, about 10 times greater than the typical duration of our experiments after nucleation. As can be seen from Fig. 11, the agreement with the quasi-equilibrium assumption is excellent when the simulation is performed over a sufficiently long time.

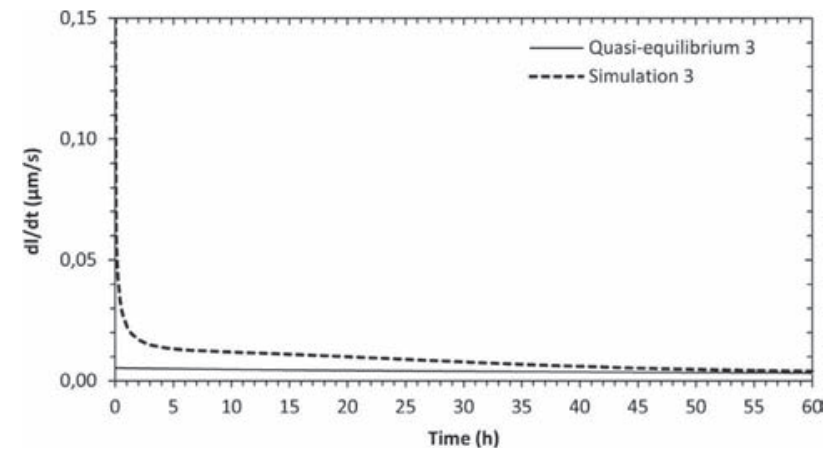

Fig. 11. Comparison between the evolution of crystal growth velocity obtained by simulation and quasi-equilibrium assumption (Eq. (17)) in case of experiment 3. $t=0$ corresponds to the onset of crystallization. 


\subsection{Crystallization pressure}

The maximum stress $\Delta P$ that can be produced from the supersaturation of a solution reads, e.g. [21]:

$\Delta P=\frac{n R T}{V_{C}} \ln S$,

where $n$ is the number of ions forming the salt (e.g. $n=2$ for $\mathrm{NaCl}$ ), $R$ is the gas constant, $T$ the temperature $(\mathrm{K}), V_{C}$ is the crystal molar volume $\left(V_{C}=2.7 \times 10^{-5} \mathrm{~m}^{3} \mathrm{~mol}^{-1}\right.$ for $\left.\mathrm{NaCl}\right)$. Applying Eqs. (18) for $S \sim 1.6$ gives $\triangle P \approx 135 \mathrm{MPA}$ which is greater than glass tensile strength $(\sim 40 \mathrm{MPa})$. However, the capillary tube is not damaged at all by the crystal growth. This can be explained as follows. First the existence of a possible stress due to supersaturation is highly transient since the supersaturation rapidly decreases in the tube (see Fig. 10). Second the crystal is actually not in contact with the tube wall when the supersaturation is high at its surface. The crystal grows in the liquid phase at a distance from the wall when the supersaturation is high. When stress can be generated on the tube wall, i.e. when the crystal is contact with the tube wall, the supersaturation has significantly decreased and the generated stress is weak, not sufficient to cause the tube rupture. In brief, these results emphasize the transient nature of the possible stress generation and that the local geometrical of the pore space shall be such that the crystal is in contact with the pore wall during the phase of high supersaturation for damages to be caused by the salt crystallization.

\section{Conclusion}

The results presented in this paper confirm that supersaturation as high as 1.6 can be expected during the evaporation of sodium-chloride solutions, at least under somewhat ideal circumstances (pure solution, carefully cleaned surfaces, minimal exposure to dirt in the environment, small volume of solution). Such a supersaturation level is sufficient to generate significant stresses on pore walls according to the known expression of crystallization pressure. However the period of possible stress generation is quite short and the growing crystal was not in contact with the tube wall when the supersaturation was high in our experiment. As a result no tube damage was observed.

This study also shows that significant ion mass fraction gradient can be generated during the evaporation process so that the consideration of average ion mass fractions cannot be sufficient to determine the supersaturation depending on the experimental conditions. This might explain in part why no supersaturation with $\mathrm{NaCl}$ was detected in some previous works, i.e. [10-13], in contrast with the result of the present study and those reported in [14]. However, it can be also argued that the situation in a porous rock or a building material is somewhat different from the ideal system considered in the present work. Building materials have heterogeneous surfaces that may favor nucleation, and the larger the body, the higher the likelihood that it contains a potent nucleating agent. This might also explain why such a level of supersaturation has not been reported so far in the available experiments with real porous media.

The study of crystal growth led to distinguish two main phases: a first, relatively short, phase where the crystal growth depends on both the precipitation reaction rate and the ion transport rate within the tube. The second phase, much longer, is characterized by comparatively low crystal growth rates and is dominated by the transport of ions in the solution and the production of ion in excess due to the liquid plug volume variations. The precipitation reaction is fast compared to the ion mass fraction variations due to transport or volume changes and therefore has no impact on the crystal growth rate in this second phase.
Also, a numerical method was described to compute the flow and the ion concentration field in the tube with a receding meniscus and a growing crystal.

Our study may help to develop a model of evaporation with salt precipitation in porous media and also to design microfluidic experiments in which the impact of crystallization pressure could be directly observed and quantified.

\section{Appendix A. Numerical solutions}

This Appendix presents the numerical solution used to compute the ion mass fraction distribution within the shrinking liquid plug during the evaporation-crystallization process. The computational domain is formed by the liquid plug and the domain occupied by the gas phase located between the tube inlet and the receding meniscus. The problem was modeled mathematically as follows assuming negligible temperature variations.

The gas phase is a water vapor-air binary mixture. The continuity equations for the gas phase and the vapor are expressed as:

$\frac{\partial \rho_{g}}{\partial t}+\rho_{g} \nabla v_{g}=0$

$\frac{\partial y_{v}}{\partial t}=D_{v} \Delta y_{v}$,

where $\rho_{g}$ is the gas density, $y_{v}$ is the vapor mass fraction, $D_{v}=2.5$ $10^{-5} \mathrm{~m}^{2} / \mathrm{s}$ is the diffusion coefficient of vapor in air, $v_{g}$ is the gas phase velocity and $t$ is the time. In fact, the problem in the gas phase can be considered as quasi-steady as in the classical one dimensional analytical approach leading to Eq. (2) in the main text. We have nevertheless solved the problem keeping the transient form of the equations. The simulation results confirmed that the evolution in the gas phase was quasi-steady.

The liquid phase is a sodium chloride aqueous solution. The continuity equation for the liquid phase, the species $(\mathrm{NaCl})$ continuity equation and the momentum balance equation are expressed as:

$\frac{\partial \rho_{\ell}}{\partial t}+\rho_{\ell} \nabla v_{\ell}=0$

$\rho_{\ell}\left[\frac{\partial \mathbf{v}_{\ell}}{\partial t}+v_{\ell} \nabla v_{\ell}\right]=-\nabla P_{\ell}+\mu_{\ell} \nabla^{2} v_{\ell}$

$\frac{\partial x_{s}}{\partial t}+\nabla \cdot\left(x_{s} v_{\ell}\right)=D_{s} \Delta x_{s}$

where $x_{s}$ is the ion mass fraction, $\rho_{\ell}$ the liquid density, $v_{\ell}$ is the liquid phase velocity, $P_{\ell}$ is the pressure in the liquid phase, $\mu_{\ell}$ is the liquid dynamic viscosity, $D_{s}=1.310^{-9} \mathrm{~m}^{2} / \mathrm{s}$ is the sodium chloride diffusion coefficient in the liquid phase.

The boundary conditions are: $y_{v}=y_{v_{\infty}}$ (which is the vapor mass fraction corresponding to the relative humidity $7 \%$ ) at the tube inlet ( $z=0$ in Fig. $1 \mathrm{~b}$ ), $v_{\ell}=0, \mathbf{v}_{g}=0, \partial x_{s} / \partial r=0$ and $\partial y_{v} / \partial r=0$ at the tube wall. The bottom meniscus is considered as a wall.

A crucial point in the model is the coupling between the gas phase and the liquid phase at the moving meniscus. The top meniscus is assumed to be a rigid interface. This assumption can be justified considering that the capillary pressure jump across the meniscus, $P_{c} \approx(4 \gamma / d)$ where $\gamma$ is the interfacial tension and $d$ the tube diameter is much greater than the pressure variation along the meniscus due to viscous dissipation. The later can be estimated as $\delta P_{\text {vis }} \approx\left(\mu_{\ell} \mathrm{v}_{m} / d\right)$ where the characteristic velocity can be estimated as the meniscus velocity:

$\mathrm{v}_{m}=\frac{d z_{m}}{d t} \approx \frac{\rho_{g}}{\rho_{\ell}} D_{v} \frac{y_{v e}-y_{v \infty}}{d}$, 
considering here an upper bound since the initial position of receding meniscus is greater than the tube diameter in the experiments. This gives $\frac{\delta P_{v i s}}{P_{c}} \approx \frac{\mu_{\ell} \rho_{g} D_{v}\left(y_{v e}-y_{v \infty}\right)}{4 \gamma \rho_{f} d} \approx 8.10^{-10}$, which justifies our assumption.

Thus, we can consider that the interface velocity, $w$, is the same at each point of the moving meniscus and can be express as $(0,0$, $w_{z}$ ) using the cylindrical coordinate system $(r, \varphi, z)$. Denoting the evaporation rate by $J$, it can be shown that:

$\frac{d z_{m}}{d t}=w_{z}=\frac{4 J}{\rho_{\ell} \pi d^{2}}$,

where $J$ can be express as:

$J=\int_{S_{m}}-\frac{\rho_{g}}{1-y_{v e}} D_{v} \nabla y_{v} n d S$.

The interfacial condition expressing the continuity of mass flux at the meniscus surface is expressed as:

$\rho_{\ell}\left(v_{\ell}-w\right) n=-\frac{\rho_{g}}{1-y_{v e}} D_{v} \nabla y_{v} n$,

As proposed by [25], the free shear stress boundary condition is expressed as:

$\mathbf{t} \cdot \tau=0$,

where $\mathbf{t}$ is a unit vector tangential to the interface and $\tau$ is the viscous stress tensor.

The assumption of local thermodynamical equilibrium is made at the meniscus. Thus,

$y_{v}=y_{v e}$.

The salt cannot cross the meniscus surface, which is expressed mathematically as:

$\left(x_{s} \rho_{\ell} v_{\ell}-\rho_{\ell} D_{s} \nabla x_{s}\right) n=\rho_{\ell} x_{s} w n$

The initial position of moving meniscus within the tube and the length of liquid plug $H$ are input data for the simulation as well as the initial ion mass fraction which is assumed spatially uniform at $t=0$.

The above problem is solved until the time where crystals are visible in the considered experiment as explained in the text.

To perform the simulation in the presence of a growing crystal at the bottom of the liquid plug, boundary conditions at the crystal-solution interface must be introduced. These conditions read:

$\rho_{\ell}\left(v_{\ell}-w_{c r}\right) n=-\rho_{c r} w_{c r} n$,

$v_{\ell} \cdot \mathbf{t}=0$,

where $\rho_{c r}$ and $w_{c r}$ are the crystal density and crystal-liquid interface velocity respectively.

$\left(x_{s} \rho_{\ell} v_{\ell}-\rho_{\ell} D_{s} \nabla x_{s}\right) n=\rho_{c r} w n$

with

$\rho_{c r} w n=K_{c r}(S-1)_{g r}$

For simplicity, it was actually assumed that the crystal-liquid interface was flat with the crystal extending over all the crosssection of the tube.

The initial conditions for the simulation considering the crystal growth are given by the results of the simulation obtained when the supersaturation marking the beginning of crystallization is reached within the tube.

The above problems were solved numerically using the commercial software Comsol Multiphysics. In order to track the interface and maintain a fine mesh near the receding meniscus or the crystal-liquid interface, a method of deformed mesh was used. We used the "deformed geometry" option proposed in the software. We checked that the salt and water mass balances were well satisfied when using this method.

Owing to the symmetry of the problem, the above problems were solved using cylindrical coordinates considering only one half of the tube longitudinal cross section.

\section{References}

[1] W.L. Olbricht, Pore-scale prototypes of multiphase flow in porous media, Annu. Rev. Fluid Mech. 28 (1) (1996) 187-213.

[2] F. Chauvet, P. Duru, S. Geoffroy, M. Prat, Three periods of drying of a single square capillary tube, Phys. Rev. Lett. 103 (12) (2009) 124502.

[3] M. Prat, Recent advances in pore-scale models for drying of porous media, Chem. Eng. J. 86 (1-2) (2002) 153-164.

[4] J. Leng, B. Lonetti, P. Tabeling, M. Joanicot, A. Ajdari, Microevaporators for kinetic exploration of phase diagrams, Phys. Rev. Lett. 96 (8) (2006) 084503.

[5] F. Hidri, N. Sghaier, H. Eloukabi, M. Prat, S. Ben Nasrallah, Porous medium coffee ring effect and other factors affecting the first crystallisation time of sodium chloride at the surface of a drying porous medium, Phys. Fluids 25 (12) (2013) 127101.

[6] S. Gupta, H.P. Huinink, M. Prat, L. Pel, K. Kopinga, Paradoxical drying of a firedclay brick due to salt crystallization, Chem. Eng. Sci. 109 (2014) 204-211.

[7] S. Veran-Tissoires, M. Prat, Evaporation of a sodium chloride solution from a saturated porous medium with efflorescence formation, J. Fluid Mech. 749 (2014) 701-749.

[8] R.M. Espinosa-Marzal, G.W. Scherer, Impact of in-pore salt crystallization on transport properties, Environ. Earth Sci. 69 (8) (2012) 2657-2669.

[9] G.W. Scherer, Stress from crystallization of salt, Cem. Concr. Res. 34 (9) (2004) $1613-1624$.

[10] L. Pel, H. Huinink, K. Kopinga, Ion transport and crystallization in inorganic building materials as studied by nuclear magnetic resonance, Appl. Phys. Lett. 81 (15) (2002) 2893.

[11] L. Pel, H. Huinink, K. Kopinga, Salt transport and crystallization in porous building materials, Magn. Reson. Imaging 21 (3-4) (2003) 317-320.

[12] L. Pel, H. Huinink, K. Kopinga, R.P.J. van Hees, O.C.G. Adan, Efflorescence pathway diagram: understanding salt weathering, Constr. Build. Mater. 18 (5) 2004) 309-313.

[13] S. Gupta, L. Pel, K. Kopinga, Crystallization behavior of $\mathrm{NaCl}$ droplet during repeated crystallization and dissolution cycles: an NMR study, J. Cryst. Growth 391 (2014) 64-71.

[14] J. Desarnaud, H. Derluyn, J. Carmeliet, D. Bonn, N. Shahidzadeh, Metastability limit for the nucleation of $\mathrm{NaCl}$ crystals in confinement, J. Phys. Chem. Lett. 5 (5) (2014) 890-895.

[15] C. Noiriel, F. Renard, M.-L. Doan, J.-P. Gratier, Intense fracturing and fracture sealing induced by mineral growth in porous rocks, Chem. Geol. 269 (3-4) (2010) 197-209.

[16] N. Shahidzadeh, J. Desarnaud, Damage in porous media: role of the kinetics of salt (re)crystallization, Eur. Phys. J. Appl. Phys. 60 (2) (2012) 24205.

[17] B. Camassel, N. Sghaier, M. Prat, S. Ben Nasrallah, Evaporation in a capillary tube of square cross-section: application to ion transport, Chem. Eng. Sci. 60 (3) (2005) 815-826.

[18] N. Sghaier, M. Prat, S. Ben Nasrallah, On the influence of sodium chloride concentration on equilibrium contact angle, Chem. Eng. J. 122 (1-2) (2006) 47-53.

[19] R.B. Bird, W.E. Stewart, E.N. Lightfood, Transport PhenomenaJohn Wiley, New York, 1960.

[20] V.M.M. Lobo, Mutual diffusion coefficients in aqueous electrolyte solutions, Pure Appl. Chem. 65 (12) (1993) 2613-2640.

[21] M. Steiger, Crystal growth in porous materials-I: the crystallization pressure of large crystals, J. Cryst. Growth 282 (3-4) (2005) 455-469.

[22] C. Buffone, K. Sefiane, J.R.E. Christy, Experimental investigation of self-induced thermocapillary convection for an evaporating meniscus in capillary tubes using micro-particle image velocimetry, Phys. Fluids 17 (5) (2005) 052104.

[23] A.E. Nielsen, J.M. Toft, Electrolyte crystal growth kinetics, J. Cryst. Growth 67 (2) (1984) 278-288.

[24] H. Derluyn, Salt Transport and Crystallization, ETH Zurich (PhD thesis), 2012.

[25] H. Hu, R.G. Larson, Analysis of the microfluid flow in an evaporating sessile droplet, Langmuir 21 (9) (2005) 3963-3971. 